\title{
A Simple Heuristic Algorithm to Determine the Set of Closed Surfaces of the Cubic Tensor Polynomial
}

\author{
Panagiotis G. Asteris* \\ Computational Mechanics Laboratory, Department of Civil \& Construction Engineering, School of Pedagogical \\ \&Technological Education, N. Heraklion, Athens, GR 14121, Greece
}

\begin{abstract}
A simple heuristic algorithm, which determines the set of closed surfaces of the cubic tensor polynomial, is proposed. The algorithm is based on the requirement of positive Gaussian curvature (Stoker's Theorem) for closed surfaces, excluding domains of tensor values, which do not provide closed surface solutions, and focus the search in the remaining domains. The identification of the closed surfaces is an important factor in both theory and applications, such as the failure behavior of anisotropic bodies. An example of applying successfully the algorithm to a material, which has a distinct anisotropic behavior, is presented.
\end{abstract}

Keywords: Cubic tensor polynomial, heuristic method, least square method, Gaussian curvature.

\section{INTRODUCTION}

In designing structural applications using composite materials it is necessary to develop methodologies for estimating the load carrying capacity under complex states of stress. One such approach is to formulate a phenomenological failure criterion for anisotropic solids, which has received considerable attention leading to a plethora of proposed criteria, the majority of which are rather limited in their capacity to explain a wide range of complex stresses [1].

Anisotropic material failure models can be grouped in three categories of increasing operational complexity. The simplest approach is to design the maximum stress or strain. However, these models lead to substantial over-estimation of strength in the 'corner' regions of the failure surface envelope. The next class of models approximates the failure surface by quadratic polynomials of different forms. Many variations of quadratic models can be found in the literature, including those that define the surface using different functions for each quadrant. Again, for certain load cases, quadratic formulations can overestimate strength [1]. Both these classes of models have the disadvantage that the failure surface is a synthesis of four surfaces at least (one for each quadrant). This leads to the emergence of singular points (that is, points of section of different surfaces) on the failure surface. For these potential surfaces the flow vector is not uniquely defined at the so-called 'corners' [2, 3] leading to indeterminate direction of straining. According to Zienkiewicz, Valliapan, and King [4], the existence of singular points imposes significant problems to the non-linear analysis. The authors propose a method to avoid singular points in a yield surface by making a suitable choice of continuous surfaces, which can represent the true condition of the anisotropic material with a good degree of accuracy. The third

*Address correspondence to this author at the Computational Mechanics Laboratory, Department of Civil \& Construction Engineering, School of Pedagogical \&Technological Education, N. Heraklion, Athens, Greece, GR 14121; Tel: +30 2102896747 ; Fax: +30 2102896788 ;

E-mail: pasteris@otenet.gr; asteris@aspete.gr category of failure models is termed 'higher order models', with the cubic polynomial [1-6] being the most common one. It should be mentioned that all these models represent approximations based on the general 'tensor polynomial' criterion advocated in Reference [1]. The single feature common to all these failure models is that they represent a phenomenological, macro-mechanics approach to predicting anisotropic materials failure.

One of the main difficulties encountered when attempting to model the failure surface with a single cubic polynomial representation is due to the mathematical nature of the cubic equation: the intrinsic complexity of the cubic function is of a magnitude, which necessitates the employment of special techniques to achieve closure of the surface.

The use of the least squares method to calculate the strength coefficients has been proposed in previous works. The successful application of the least squares method requires a plethora of experimental data on the all quadrants, a requirement which is expensive and not always feasible.

For example, in the case of brittle materials such as masonry, the development of such an extensive experimental data base in the tension-tension quadrant is too difficult to be fulfilled. The absence of experimental data in a quadrant leads very often to the derivation of an open surface. To overcome the problem of 'openness' (non-closure) of the failure surface, a new approach is being proposed based on the heuristic method, which leads to closure of the cubic polynomial surface and estimates the best fit to the experimental data. The proposed approach has been applied to the masonry anisotropic material.

\section{MATHEMATICAL FRAMEWORK}

The most general failure criterion available for anisotropic materials is the tensor polynomial, which was advocated as early as 1966 by Malmeister [7] and developed extensively by Tsai and $\mathrm{Wu}$ [1] and Jiang and Tennyson [6] in quadratic and higher order forms. The failure surface in the stress space, can be described by the equation 


$$
f(\sigma)=F_{i} \sigma_{i}+F_{i j} \sigma_{i} \sigma_{j}+F_{i j k} \sigma_{i} \sigma_{j} \sigma_{k}+\cdots\left\{\begin{array}{l}
\langle 1 \text { no failure } \\
=1 \text { failure } \\
>1 \text { exceeded failure }
\end{array}\right.
$$

for $\mathrm{i}, \mathrm{j}, \mathrm{k}=1,2, \ldots, 6 . F_{i}, F_{i j}$ and $F_{i j k}$ are (strength) tensors of the second, fourth and sixth rank, respectively.

If one restricts the analysis to a plane stress state and considers that a cubic formulation is a reasonably accurate representation of the failure surface, making assumptions based on the symmetry and anisotropic nature of the material $[6,8]$, Equation 1 reduces to:

$$
\begin{aligned}
f\left(\sigma_{1}, \sigma_{2}, \sigma_{6}\right)= & F_{1} \sigma_{1}+F_{2} \sigma_{2}+F_{11} \sigma_{1}^{2}+F_{22} \sigma_{2}^{2}+F_{66} \sigma_{6}^{2}+2 F_{12} \sigma_{1} \sigma_{2}+3 F_{112} \sigma_{1}^{2} \sigma_{2} \\
& +3 F_{122} \sigma_{1} \sigma_{2}^{2}+3 F_{166} \sigma_{1} \sigma_{6}^{2}+3 F_{266} \sigma_{2} \sigma_{6}^{2}-1=0
\end{aligned}
$$

This general form of the cubic tensor failure criterion can be shown that encompasses all other failure criteria, which are currently available [9-12].

The problem that one is confronted with is the evaluation of the strength tensors. For the solution, basic principles and tools should be formulated.

\section{Basic Principle}

The failure surface of the cubic function of Equation (2) must be a closed one (closure) due to the fact that, in nature, for each possible stress state the material is failed under finite values of stress vector; no material is indestructible. In mathematical terms, this basic principle can be expressed by the following Stoker's uniqueness theorem for closed surfaces in three-space $[13,14]$ :

\section{Stoker's Theorem}

A surface is closed if the total Gaussian curvature $\mathrm{K}$ at any points of the surface is positive.

It is to be noted that the sign of $\mathrm{K}$ fixes in a qualitative way the shape of a surface near a given point. In geometric terms, if the $\mathrm{K}$ of a surface point is positive, the point is elliptic.

According to Mishchenko, Solovyev, Fomenko [15], the total Gaussian curvature $\mathrm{K}$ for the surface given by the Equation 2 is given by

$$
K=-\frac{1}{\left(\partial f / \partial \sigma_{1}\right)^{2}+\left(\partial f / \partial \sigma_{2}\right)^{2}+\left(\partial f / \partial \sigma_{6}\right)^{2}} D
$$

As the denominator is always positive, the total Gaussian curvature $\mathrm{K}$ of the proposed surface is positive if:

$$
D=\left|\begin{array}{cccc}
\partial^{2} f / \partial \sigma_{1} \partial \sigma_{1} & \partial^{2} f / \partial \sigma_{1} \partial \sigma_{2} & \partial^{2} f / \partial \sigma_{1} \partial \sigma_{6} & \partial f / \partial \sigma_{1} \\
\partial^{2} f / \partial \sigma_{1} \partial \sigma_{2} & \partial^{2} f / \partial \sigma_{2} \partial \sigma_{2} & \partial^{2} f / \partial \sigma_{2} \partial \sigma_{6} & \partial f / \partial \sigma_{2} \\
\partial^{2} f / \partial \sigma_{1} \partial \sigma_{6} & \partial^{2} f / \partial \sigma_{2} \partial \sigma_{6} & \partial^{2} f / \partial \sigma_{6} \partial \sigma_{6} & \partial f / \partial \sigma_{6} \\
\partial f / \partial \sigma_{1} & \partial f / \partial \sigma_{2} & \partial f / \partial \sigma_{6} & 0
\end{array}\right|<0
$$

Lemma 1: Let

$$
f\left(\sigma_{1}, \sigma_{2}, \sigma_{6}\right)=F_{1} \sigma_{1}+F_{2} \sigma_{2}+F_{11} \sigma_{1}^{2}+F_{22} \sigma_{2}^{2}+F_{66} \sigma_{6}^{2}+2 F_{12} \sigma_{1} \sigma_{2}-1=0
$$

be an equation of the second degree (the corresponding quadratic form of Equation 2), which is not factorable. Its graph is always a closed surface if

$$
-\sqrt{F_{11} F_{22}}<F_{12}<\sqrt{F_{11} F_{22}}
$$

\section{Proof}

The surface will be closed if any curve for varying $\sigma_{6}$ is closed. Thus, according to Stocker's Theorem and for the case of function $\mathrm{f}$ of $\mathrm{Eq}$ (5), Equation 4 becomes

$D=\left|\begin{array}{ll}\partial^{2} f / \partial \sigma_{1} \partial \sigma_{1} & \partial^{2} f / \partial \sigma_{1} \partial \sigma_{2} \\ \partial^{2} f / \partial \sigma_{1} \partial \sigma_{2} & \partial^{2} f / \partial \sigma_{2} \partial \sigma_{2}\end{array}\right|<0$

(because all other second derivatives are 0 , except the $\partial^{2} f / \partial \sigma_{6} \partial \sigma_{6}$ term which is the positive coefficient $F_{66}$ ).

For the case of the function $f$ of Eq. (5), the denominator D of Eq. (7) leads to the proof of Lemma 1.

In order to achieve closure of the surface, Tennyson \& Elliot [16], have studied the quadratic model with $F_{12}=0$ and $F_{12}=-\frac{1}{2} \sqrt{F_{11} F_{22}}$; both these values belong to the proposed range of values (Inequality 6) within which the set of closed surfaces resides.

\section{EVALUATING THE TENSORS}

The determination of the strength tensors is made in two steps. In the first step, the determination of Principal Strength Tensor Components $\left(F_{i}\right.$ and $F_{i i}$ ) is taking place. In the second step, the determination of the Interaction Strength Tensor Components ( $F_{i j}$ and $F_{i j k}$ ) occurs.

\section{First Step}

It has been shown [5] that the Principal Strength Tensors $\left(F_{i}\right.$ and $F_{i i}$ ) can be readily calculated from the experimentally determined values of the uniaxial tensile and compressive strengths along the longitudinal ( $X$ and $X^{\prime}$ ) and the transverse directions ( $Y$ and $Y^{\prime}$, respectively) and from positive and negative pure shear strengths ( $S$ and $S^{\prime}$, respectively) in the principal directions. These uniaxial stresses define the six boundary points of tensor polynomial surface, namely the $(X, 0,0),\left(-X^{\prime}, 0,0\right),(0, Y, 0),\left(0,-Y^{\prime}, 0\right),(0,0, S)$, and $\left(0,0,-S^{\prime}\right)$, points. For these boundary point values the appropriate relations of the Principal strength tensors are given by [5]:

$$
\begin{array}{ll}
F_{1}=\frac{1}{X}-\frac{1}{X^{\prime}}, & F_{11}=\frac{1}{X X^{\prime}} \\
F_{2}=\frac{1}{Y}-\frac{1}{Y^{\prime}}, & F_{22}=\frac{1}{Y Y^{\prime}} \\
F_{66}=\frac{1}{S^{2}} &
\end{array}
$$

\section{Second Step}

The problem that one is confronted with is the evaluation of the remaining interaction Strength Tensor Components $\left(F_{i j}\right.$ and $\left.F_{i j k}\right)$. It has been proposed that the values of these parameters can be calculated using the least squares method 
[5-6, 8]. The minimum value of $f$ yielding a 'best' least squares fit is obtained from

$\frac{\partial E_{v}}{\partial \mathrm{F}_{12}}=0, \quad \frac{\partial E_{v}}{\partial \mathrm{F}_{112}}=0, \quad \frac{\partial E_{v}}{\partial \mathrm{F}_{122}}=0, \quad \frac{\partial E_{v}}{\partial \mathrm{F}_{166}}=0, \quad \frac{\partial E_{v}}{\partial \mathrm{F}_{266}}=0$

where:

$$
\begin{aligned}
E_{v}=\sum_{i=1}^{v}\left(F_{1} \sigma_{1 i}\right. & +F_{2} \sigma_{2 i}+F_{11} \sigma_{1 i}^{2}+F_{22} \sigma_{2 i}^{2}+F_{66} \sigma_{6 i}^{2}+2 F_{12} \sigma_{1 i} \sigma_{2 i}+ \\
& \left.+3 F_{112} \sigma_{1 i}^{2} \sigma_{2 i}+3 F_{166} \sigma_{1 i} \sigma_{6 i}^{2}++3 F_{266} \sigma_{2 i} \sigma_{6 i}^{2}-1\right)^{2}
\end{aligned}
$$

with $v$ denoting the number of the experimental sets of values $\left(\sigma_{1 i}, \sigma_{2 i}, \sigma_{6 i}\right)(\mathrm{i}=1,2, \ldots, v)$.

Solving the Equation 9, the (remaining) parameters $F_{12}$, $F_{112}, F_{122}, F_{166}$ and $F_{266}$ can be determined, representing the 'best fit' to experimental data. However, the solution does not correspond always to a closed surface. By not being able to achieve, many researchers are either employing semiempirical methods [16] or reducing the number of independent parameters [17].

Our proposed approach reveals the set of closed surfaces (identifying the best fit solution to the experimental data) without constraining its approach to semi-empirical methods or reducing the number parameters.

\section{PROPOSED HEURISTIC ALGORITHM}

To overcome the problem of 'openness' (non-closure) of the failure surface, a new approach is being proposed, based on heuristic methods, which satisfies the closure of the cubic polynomial surface and provides the best fit to the experimental data. The proposed approach is implemented by applying the following algorithm:

\section{Step 1}

Set the first value of the parameter $F_{12}$ as $F_{12}(i=1)=-\sqrt{F_{11} F_{22}}$, The choice of this value stems from Lemma 1; it constitutes the left limit of the inequality $-\sqrt{F_{11} F_{22}}<F_{12}<\sqrt{F_{11} F_{22}}$. Beyond the limits of this interval, only open surfaces will occur.

\section{Step 2}

Having setting up the value of $F_{12}(i)$, the remaining parameters $F_{112}(i), F_{122}(i), F_{166}(i)$ and $F_{266}(i)$ are calculated by solving the system of equations (9).

\section{Step 3}

For the obtained values of the parameters $F_{12}(i)$, $F_{112}(i), F_{122}(i), F_{166}(i)$ and $F_{266}(i)$ :

3.1 calculate the deviation $E_{v}$ (equation 10) of the corresponding surface to the experimental data, and

3.2 check if the corresponding surface complies to the requirement of positive total curvature (Equation 4), that is, the surface is closed.

\section{Step 4}

Set $F_{12}(i+1)=F_{12}(i)+\Delta F_{12}$, where $\Delta F_{12}=2 \sqrt{F_{11} F_{22}} / n$ with $n=10$ (a mesh size, which is sufficient for the case of engineering materials). Iterate through Steps 2, 3, and 4. If $\left.F_{12}(i+1)\right\rangle \sqrt{F_{11} F_{22}}$ then stop.

From all closed surfaces, the one with the smallest deviation corresponds to the best fit to the available experimental data. However, the practicing engineer may select a closed solution, which, based on his/her experience, may represent a more optimal solution to the problem at hand.

\section{EXAMPLE}

In the following example the proposed algorithm is applied to define the failure surface in the case of a masonry material, the behavior of which has been well studied experimentally [18].

The experimental values for the monoaxial failure strength estimated from graphs [18] are $\mathrm{X}=0.40 \mathrm{MPa}, \mathrm{X}^{\prime}=4.3625$ $\mathrm{MPa}, \mathrm{Y}=0.10 \mathrm{MPa}, \mathrm{Y}^{\prime}=7.555 \mathrm{MPa}, \mathrm{S}=\mathrm{S}^{\prime}=0.40 \mathrm{MPa}$.

With these values on equations 8 , the tensors $F_{i}, F_{i i}$ become: $\mathrm{F}_{1}=0.227 \mathrm{E}+01 \quad(\mathrm{MPa})^{1}, \quad \mathrm{~F}_{11}=0.573 \mathrm{E}+00 \quad(\mathrm{MPa})^{-2}$, $\mathrm{F}_{2}=0.987 \mathrm{E}+01 \quad(\mathrm{MPa})^{-1}, \quad \mathrm{~F}_{22}=0.132 \mathrm{E}+01 \quad(\mathrm{MPa})^{-2}$, $\mathrm{F}_{66}=0.625 \mathrm{E}+01(\mathrm{MPa})^{-2}$.

In order to determine constants $F_{12}, F_{112}, F_{122}, F_{166}$ and $F_{266}$, the equation 10 is solved using the experimental data

Table 1. Summary of Test Data Used to Evaluate the Interaction Strength Tensor Parameters for Masonry Material

\begin{tabular}{|c|c|c|c|}
\hline Test No & $\begin{array}{c}\sigma_{1} \\
(\mathbf{M P a})\end{array}$ & $\begin{array}{c}\sigma_{2} \\
(\mathbf{M P a})\end{array}$ & $\begin{array}{c}\sigma_{6} \\
(\mathbf{M P a})\end{array}$ \\
\hline \hline 1 & -0.727 & -7.542 & 0.000 \\
2 & -0.727 & -8.417 & 0.000 \\
3 & -2.272 & -9.250 & 0.000 \\
4 & -2.181 & -8.750 & 0.000 \\
5 & -4.545 & -8.667 & 0.000 \\
6 & -7.909 & -7.791 & 0.000 \\
7 & -8.818 & -8.750 & 0.000 \\
8 & -9.454 & -4.792 & 0.000 \\
9 & -9.590 & -2.333 & 0.000 \\
10 & -11.273 & -5.583 & 0.000 \\
11 & -9.272 & -1.000 & 0.000 \\
12 & -4.181 & -8.000 & 0.000 \\
13 & -9.909 & -5.042 & 0.000 \\
14 & -8.308 & -8.475 & 0.084 \\
15 & -4.555 & -1.310 & -1.622 \\
16 & -5.821 & -5.821 & 3.571 \\
17 & -6.620 & -6.620 & 2.120 \\
18 & -5.821 & -5.821 & -3.571 \\
19 & -6.620 & -6.620 & -2.120 \\
20 & -8.273 & -8.475 & -0.084 \\
21 & -5.227 & -1.310 & 1.622 \\
22 & -4.181 & -8.000 & 0.000 \\
23 & -9.909 & -5.042 & 0.000 \\
\hline
\end{tabular}


of Table 1. Their values are: $F_{12}=1.640209 \mathrm{E}-02(\mathrm{MPa})^{-2}$, $\mathrm{F}_{112}=9.453948 \mathrm{E}-03 \quad(\mathrm{MPa})^{-3}, \quad \mathrm{~F}_{122}=8.886827 \mathrm{E}-03 \quad(\mathrm{MPa})^{-3}$, $\mathrm{F}_{166}=1.365468 \mathrm{E}-01(\mathrm{MPa})^{-3}, \mathrm{~F}_{266}=1.2929976 \mathrm{E}-01(\mathrm{MPa})^{-3}$.

The surface corresponding to these values should be checked for closed form. The surface (see Fig. 1) is open because its total curvature is negative (condition 12 is not valid in this case), as is often the case.

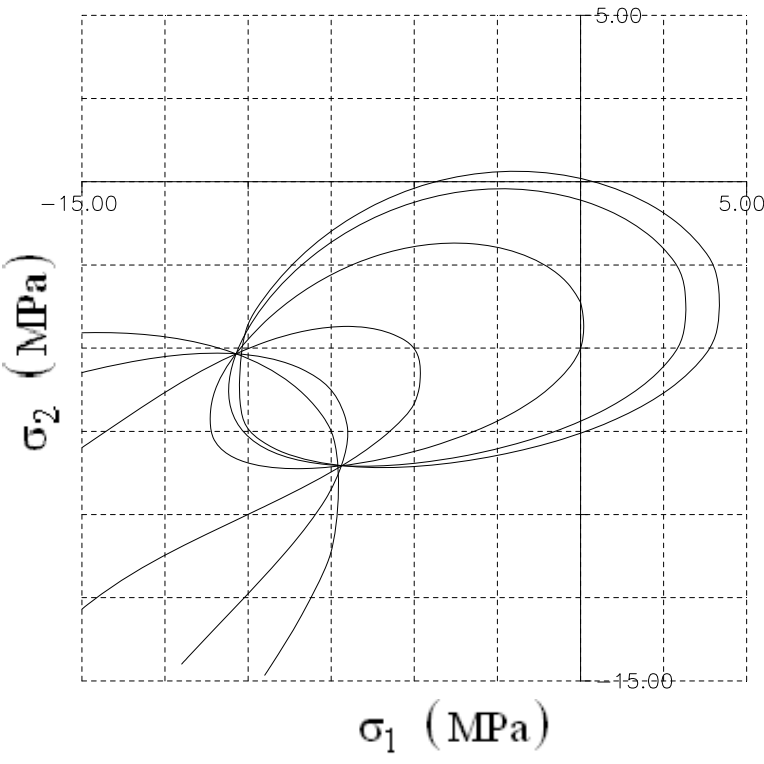

Fig. (1). Open failure surface contours $\left(\sigma_{6}=0.00\right.$ up to 5.00 by step=1.00).

The algorithm proceeds and identifies the range of values of tensor F12, which correspond to closed surfaces as:

$-0.710 \leq F_{12} \leq-0.150$

In this range of values, the best-fit solution (which is least deviated from the experimental data) corresponds to $F_{12}=-0.150(\mathrm{MPa})^{-2}$. For this value, the other tensors become $\mathrm{F}_{112}=0.3195 \mathrm{E}-02 \quad(\mathrm{MPa})^{-3}, \quad \mathrm{~F}_{122}=0.1045 \mathrm{E}-02 \quad(\mathrm{MPa})^{-3}$, $\mathrm{F}_{166}=0.9466 \mathrm{E}-01(\mathrm{MPa})^{-3}, \mathrm{~F}_{266}=0.1563 \mathrm{E}+00(\mathrm{MPa})^{-3}$.

Thus, the failure surface (Fig. 2) for the masonry material is described by the equation:

$$
\begin{aligned}
2.27 \sigma_{1} & +9.87 \sigma_{2}+0.573 \sigma_{1}^{2}+1.32 \sigma_{2}^{2}+6.25 \sigma_{6}^{2}-0.30 \sigma_{1} \sigma_{2}+ \\
& +0.009585 \sigma_{1}^{2} \sigma_{2}+0.003135 \sigma_{1} \sigma_{2}^{2}+0.28398 \sigma_{1} \sigma_{6}^{2}+0.4689 \sigma_{2} \sigma_{6}^{2}=1
\end{aligned}
$$

\section{CONCLUSIONS}

In the present paper, an algorithm to determine the closed surfaces of the cubic tensor polynomial is proposed. From all closed surfaces, the one with the smallest deviation corresponds to the best fit to the available experimental data. However, the practicing engineer may select a closed solution, which, based on his/her experience may represent a more optimal solution to the problem at hand. Our algorithm may offer significant support in areas such as the research on composite laminates, and the failure of trabecular bone in

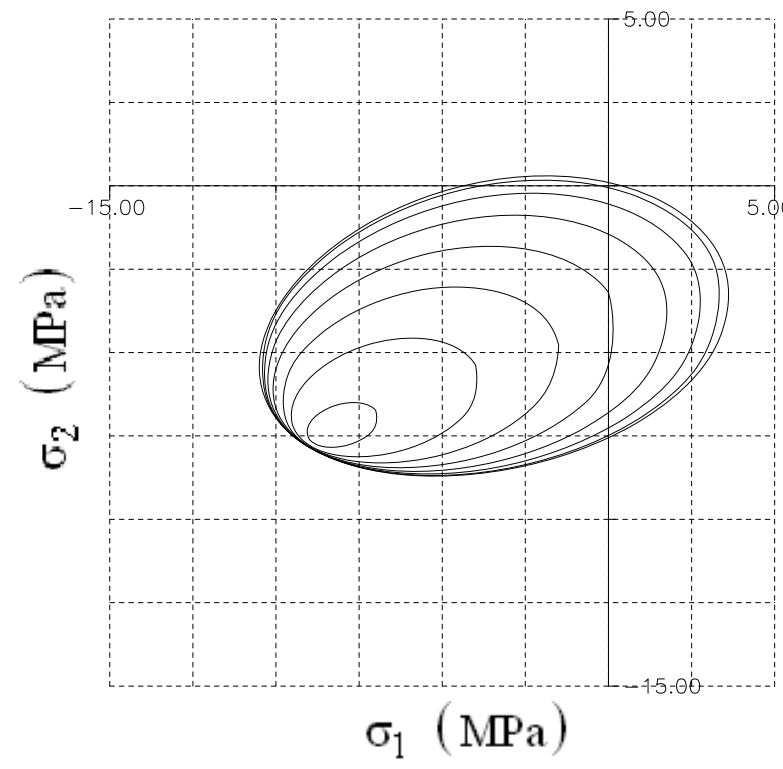

Fig. (2). Closed failure surface contours for masonry material ( $\sigma_{6}=0.00$ up to 3.50 by step $=0.50$ ).

many biomechanical applications (facilitating the investigation of the effects of aging, disease, and drug treatment), in addition to the research on masonry materials.

\section{REFERENCES}

[1] Tsai SW, Wu EM. A general failure criterion for anisotropic materials. J Comp Mat 1971; 5: 58-80.

[2] Koiter WT. Stress-strain relations, uniqueness and variational theorems for elastic-plastic materials with singular yield surface. Q Appl Meth 1953; 11: 350-54.

[3] Bland DR. The associated flow rule of plasticity. J Mech Phys Solids 1957; 6: 71-8.

[4] Zienkiewicz OC, Valliapan S, King IP. Elasto-plastic solutions of engineering problems; Initial stress finite element approach. Int J Num Meth Eng 1969; 1: 75-100.

[5] Wu EM. Optimal experimental measurements of anisotropic failure tensors. J Comp Mat 1972; 6: 472-80.

[6] Jiang Z, Tennyson RC. Closure of the cubic tensor polynomial failure surface. J Comp Mat 1989; 23: 208-31.

[7] Malmeister AK. Geometry of theories of strength. Mekhanika Polimerov 1966; 2(4): 519-534.

[8] Syrmakezis CA, Asteris PG. Masonry failure criterion under biaxial stress state. J Mat Civil Eng (ASCE) 2001; 13(1): 58-64.

[9] Arramon YP, Mehrabadi MM, Martin DW. A multidimensional anisotropic strength criterion based on Kelvin modes. Int J Solids Struct 2000; 37: 2915-35.

[10] Christensen RM, Freeman DC, DeTeresa SJ. Failure criteria for isotropic materials, applications to low-density types. Int J Solids Struct 2002; 39: 973-82.

[11] Hinton MJ, Kaddour AS, Soden PD. A comparison of the predictive capabilities of current failure theories for composite laminates, judged against. Comp Sci Technol 2002; 62: 1725-97.

[12] Christensen RM, DeTeresa SJ. Delamination failure investigation for out-of-plane loading in laminates. J Comp Mat 2004; 38(24): 2231-38.

[13] Stoker JJ. Differential geometry. NewYork: Wiley 1969.

[14] Stoker JJ. Uniqueness theorems for some open and closed surfaces in three-space. Annali della Scuola Normale Superiore di Pisa Classe di Scienze, Sér. 4, 1978; 5 (4): 657-77.

[15] Mishchenko AS, Solovyev YP, Fomenko AT. Problems in differential geometry and topology. Translated from the Russian by Oleg Efimov. Moscow: Mir Publishers 1985.

[16] Tennyson RC, Elliot WG. Development of failure criterion for Kevlar-Epoxy fabric laminates. NASA CR 172465, 1984. 
[17] DeTeresa SJ, Larsen GJ. Reduction in the number of independent parameters for the tsai-wu tensor polynomial theory of strength for composite materials. J Comp Mat 2003; 37(19): 1769-85.
[18] Page AW. The biaxial compressive strength of brick masonry. Proc Inst Civil Eng 1981; 2(71): 893-906.

(C) Panagiotis G. Asteris; Licensee Bentham Open.

This is an open access article licensed under the terms of the Creative Commons Attribution Non-Commercial License (http://creativecommons.org/licenses/by-nc/3.0/) which permits unrestricted, non-commercial use, distribution and reproduction in any medium, provided the work is properly cited. 